\title{
Caracterização genética de um teste de progênies de Dipteryx alata Vog. proveniente de remanescente florestal da Estação Ecológica de Paulo de Faria, SP, Brasil
}

\author{
Daniela Silvia de Oliveira Canuto ${ }^{1}$, Darlin Ulises Gonzalez Zaruma², Marcela Aparecida de Moraes ${ }^{1}$, \\ Alexandre Marques da Silva ${ }^{1}$, Mario Luiz Teixeira de Moraes ${ }^{1}$ e Miguel Luiz Menezes Freitas ${ }^{3,4}$
}

Recebido: 30.04.2015; aceito: 15.09.2015

\begin{abstract}
Genetic characterization of a progeny test of Dipteryx alata Vog., from a forest fragment of Estação Ecológica Paulo de Faria, São Paulo State, Brazil). Currently, the natural vegetation of the Cerrado is highly fragmented, causing the loss of genetic variability and concomitant risk of extinction of the remaining populations in these fragments. Not indifferent to this, the aim of this study was to estimate the genetic variability of a progeny test of Dipteryx alata, formed from the material of Estação Ecológica Paulo de Faria, to effect the genetic conservation of what remains of the species in the State of São Paulo and subsidize a selection method aimed the formation of a seed orchard with genetic quality to be used in the Cerrado restoration. The progeny test, with 59 progenies, 17 replications and a plant per plot, was installed on July 10, 2013, in the Fazenda de Pesquisa, Ensino e Extensão (FEPE) of Universidade Estadual Paulista, Ilha Solteira, SP, Brazil. The genetic parameter estimates were based on total plant height of 3, 7, and 21 months of age. Estimates of average heritability, individual genetic variation coefficient, accuracy $(0.81,39.88$, and 0.90 , respectively, at three months), and genetic correlations between ALT and DMC $(0.74)$ indicated that the formation of the test progenies allowed to rescue the genetic material with enough genetic variability to ensure the formation of new populations and estimates of genetic parameters showed promising genetic basis, which allows gains in selection in breeding programs and seed orchard formation for use in restoration programs.
\end{abstract}

Keywords: baru, forest genetic resources, genetic improvement, seed production

RESUMO - (Caracterização genética de um teste de progênies de Dipteryx alata Vog., proveniente de remanescente florestal da Estação Ecológica de Paulo de Faria, SP, Brasil). Atualmente a vegetação natural do Cerrado encontra-se altamente fragmentada, causando a perda da variabilidade genética e, concomitantemente, risco de extinção das populações remanescentes nestes fragmentos. Não indiferente a isto, o objetivo desse trabalho foi estimar a variabilidade genética de um teste de progênies de Dipteryx alata, formado a partir do material da Estação Ecológica de Paulo de Faria, para efetivar a conservação genética do que ainda resta da espécie no Estado de São Paulo e subsidiar um método de seleção visando a formação de pomar de sementes com qualidade genética para serem empregadas na restauração do Cerrado. O teste de progênies, composto por 59 progênies, 17 repetições e uma planta por parcela, foi instalado no dia 10 de julho de 2013, na Fazenda de Ensino, Pesquisa e Extensão (FEPE) da Universidade Estadual Paulista, Ilha Solteira, SP, Brasil. As estimativas dos parâmetros genéticos foram baseadas na altura total de plantas com 3,7 e 21 meses de idade. As estimativas de herdabilidade média, coeficiente de variação genética individual, acurácia $(0,81,39,88$ e 0,90 , respectivamente, aos três meses) e correlações genéticas entre ALT e DMC $(0,74)$ indicaram que a formação do teste de progênies permitiu resgatar o material genético com variabilidade genética suficiente para garantir a formação de novas populações e as estimativas dos parâmetros genéticos apresentaram base genética promissora, o que permite ganhos na seleção em programas de melhoramento e a formação de pomar de sementes para utilização em programas de restauração.

Palavras-chave: baru, melhoramento genético, produção de sementes, recursos genéticos florestais

\section{Introdução}

O Cerrado, depois da Mata Atlântica, é o bioma brasileiro que mais sofreu alterações com a ocupação humana. Com a crescente pressão para a abertura de novas áreas, visando incrementar a produção de carne e grãos para exportação, tem havido um progressivo esgotamento dos recursos naturais da região. Nas três

1. Universidade Estadual Paulista, Faculdade de Engenharia de Ilha Solteira, Avenida Brasil 56, 15385-000 Ilha Solteira, SP, Brasil

2. Universidade Estadual Paulista, Faculdade de Ciências Agronômicas, Fazenda Lageado, Rua José Barbosa de Barros 1780, 18.610-307 Botucatu, SP, Brasil

3. Instituto Florestal, Rua do Horto 931, 02377-000 São Paulo, SP, Brasil

4. Autor para correspondência: miguellmfreitas@yahoo.com.br 
últimas décadas, o Cerrado vem sendo degradado pela expansão da fronteira agrícola brasileira. Além disso, o Cerrado é palco de uma exploração extremamente predatória de seu material lenhoso para produção de carvão. Além dos aspectos ambientais, o Cerrado tem grande importância social, muitas populações sobrevivem de seus recursos naturais, mais de 10 tipos de frutos comestíveis são regularmente consumidos pela população local e vendidos nos centros urbanos, e dentre esses estão as sementes de baru (Dipteyx alata Vog.) (MMA 2015).

Dipteryx alata pertence à família Fabaceae e é conhecido principalmente por sua amêndoa que tem elevada importância, pois é uma rica fonte alimentar alternativa, já que essa semente possui $29 \%$ de proteína, $40 \%$ de lipídios, $19 \%$ de fibra total e 7,28\% de açúcares totais (Togashi 1993). A polpa do fruto pode ser aproveitada como ração animal, bem como fertilizantes; a semente pode ser empregada no balanço de ração dietética (Vallilo et al. 2001) e o óleo da semente pode ser usado para fins comestíveis ou como matéria-prima para indústria farmacêutica e oleoquímica (Takemoto et al. 2001). Possui mercado expressivo no Estado de Goiás e grande potencial produtivo no Cerrado (Vera et al. 2009). No Estado de São Paulo, quase não existem populações nativas de Dipteryx alata para efeito da implantação de um programa de conservação e melhoramento genético. No entanto, ainda restam alguns remanescentes no extremo norte paulista (Durigan et al. 2007).

Para justificar a conservação e uso de uma espécie é necessária a caracterização genética e morfológica, bem como desenvolvimento de técnicas silviculturais para garantir o sucesso da conservação ex situ, principalmente os realizados em áreas degradadas. Não só o comportamento, mas a base genética é uma peça chave para o sucesso dos plantios em áreas perturbadas a longo prazo. Para conservação genética de uma espécie, tem-se sempre a preocupação em manter o máximo de sua variabilidade genética existente em suas populações naturais para possibilitar a continuidade da evolução (Frakel \& Soule 1981). Portanto, se todos os reflorestamentos fossem implantados com sementes ou mudas advindas de populações naturais ou coleções de germoplasma com qualidade genética, a conservação da espécie seria mais eficiente.

Os testes de progênies atendem várias finalidades importantes para conservação das espécies: conservar a variabilidade genética de uma população natural, principalmente as populações que apresentam mais riscos de serem extintas; viabilizar a caracterização genética da espécie, por apresentar delineamentos experimentais adequados; e formar pomares de sementes por mudas em diversos sítios, viabilizando a formação de raças locais, consequentemente aumentando a taxa de sobrevivência das espécies. Esses testes constituem-se em um plantio sistematizado, onde cada árvore mantém sua referência de origem da população e possui repetições suficientes para se estimar os parâmetros genéticos, como os coeficientes de variação genético, as herdabilidades, a acurácia e ganhos de seleção, assim sendo esse material genético é conservado e melhorado.

Sendo assim, o objetivo desse trabalho foi estimar a variabilidade genética de um teste de progênies de Dipteryx alata, formado a partir do material da Estação Ecológica de Paulo de Faria, para efetivar a conservação genética do que ainda resta da espécie no Estado de São Paulo e subsidiar um método de seleção visando à formação de pomar de sementes com qualidade genética para serem empregadas na restauração do Cerrado.

\section{Material e métodos}

Em setembro de 2012 foi feita a coleta de frutos de Dipteryx alata em 59 árvores (matrizes), aleatoriamente, de polinização livre, com distância de pelo menos 100 m uma da outra, na Estação Ecológica de Paulo de Faria, pertencente ao Instituto Florestal de São Paulo, localizada no município de Paulo de Faria, SP, Brasil. A descrição detalhada da vegetação que ocorre nesta estação é citada por Stranghetti \& Ranga (1998).

As sementes foram levadas à Faculdade de Engenharia de Ilha Solteira, da Universidade Estadual Paulista, onde foram beneficiados aproximadamente 40 frutos por árvore coletada. No final de novembro e início de dezembro de 2012, as sementes foram semeadas no viveiro de mudas na Fazenda de Ensino, Pesquisa e Extensão (FEPE), localizada em Selvíria, MS, nas coordenadas geográficas $51^{\circ} 22^{\prime} \mathrm{W}$ e $2022^{\prime} \mathrm{S}$ e altitude aproximada de $335 \mathrm{~m}$.

O clima da região é do tipo Aw, definido como tropical úmido com estação chuvosa no verão e seca no inverno, segundo a classificação internacional de Köeppen, apresentando temperatura, precipitação e umidade relativa média anual de $24,5^{\circ} \mathrm{C}, 1.370 \mathrm{~mm}$ e $64,8 \%$, respectivamente (Hernandez et al. 1995). O solo da área experimental foi previamente classificado como Latossolo Vermelho distrófico 
típico, muito argiloso, A moderado, hipodistrófico, álico, caulinitico, férrico, epicompactado, muito profundo e moderadamente ácido (EMBRAPA, 1999).

Aos sete meses de idade das mudas, o teste de progênies de Dipteryx alata foi instalado no dia 10 de julho de 2013, na FEPE. Trata-se de um plantio puro, mas em sistema silvipastoril, com duas linhas de 1,5 ×3,0 m espaçada por 4,5 m. O delineamento experimental foi o de blocos completos casualizados, com 59 progênies, 17 repetições e uma planta por parcela. Foram avaliados a altura total de plantas (m) aos três, sete e 21 meses de idade, com auxílio de régua graduada em centímetros; o diâmetro médio do caule na altura do solo (DMC, $\mathrm{cm}$ ), aos sete meses de idade após o plantio; e a sobrevivências de plantas (\%) aos 21 meses, esta com a finalidade de avaliar a adaptação das plantas no local, sem a intenção de realizar seleção baseado na sobrevivência.

As estimativas de componentes de variância e parâmetros genéticos foram obtidas para os caracteres altura e DMC, pelo método Reml/ Blup (máxima verossimilhança restrita/melhor predição linear não viciada), empregando-se o software genético-estatístico Selegen-Reml/ Blup, desenvolvido por Resende (2007a). Pela metodologia do modelo linear misto (aditivo univariado) - Reml/Blup, aplicado aos testes de progênies de polinização aberta (assumindo progênies de meios-irmãos), delineamento em blocos ao acaso, uma planta por parcela, seguindo o procedimento proposto por Resende (2002 e 2007b): $\mathrm{y}=\mathrm{Xr}+\mathrm{Za}+e$; em que: y é o vetor de dados; $\mathrm{r}$ é $o$ vetor dos efeitos de repetição (assumidos como fixos) somados à média geral; a é o vetor dos efeitos genéticos aditivos individuais (assumidos como aleatórios), e $e$ é o vetor de erros ou resíduos (aleatórios). As letras maiúsculas representam as matrizes de incidência para os referidos efeitos.

Os parâmetros genéticos estimados foram:

a) Variância genética aditiva $\left(\hat{\sigma}_{a}^{2}\right)$

b) Variância residual $\left(\hat{\sigma}_{e}^{2}\right)$

c) Variância fenotípica individual $\left(\hat{\sigma}_{f}^{2}\right)$ :

$\hat{\sigma}_{f}^{2}=\hat{\sigma}_{a}^{2}+\hat{\sigma}_{c}^{2}+\hat{\sigma}_{e}^{2}$

d) Herdabilidade individual no sentido restrito, ou seja, dos efeitos aditivos $\left(\hat{h}_{a}^{2}\right)$ :

$\hat{h}_{a}^{2}=\frac{\hat{\sigma}_{a}^{2}}{\hat{\sigma}_{f}^{2}}$ e) Herdabilidade da média de progênies, assumindo sobrevivência completa $\left(\hat{h}_{m}^{2}\right)$ :

$$
\hat{h}_{m}^{2}=\frac{(1 / 4) \cdot \hat{\sigma}_{a}^{2}}{(1 / 4) \cdot \hat{\sigma}_{a}^{2}+\frac{\hat{\sigma}_{c}^{2}}{r}+\frac{\left(0,75 \cdot \hat{\sigma}_{a}^{2}+\hat{\sigma}_{e}^{2}\right)}{n \cdot r}}
$$

f) Herdabilidade aditiva dentro de parcela $\left(\hat{h}_{a d}^{2}\right)$ :

$$
\hat{h}_{a d}^{2}=\frac{0,75 \cdot \hat{\sigma}_{a}^{2}}{0,75 \cdot \hat{\sigma}_{a}^{2}+\hat{\sigma}_{e}^{2}}
$$

g) Coeficiente de variação genética aditiva individual (evolvabilidade, Houle 1992) $\left(C V_{g i}\right)$ :

$$
C V_{g i}(\%)=\frac{\sqrt{\hat{\sigma}_{a}^{2}}}{\hat{m}} \cdot 100
$$

h) Coeficiente de variação genotípica entre progênies $\left(C V_{g p}\right)$ :

$$
C V_{g p}(\%)=\frac{\sqrt{0,25 \cdot \hat{\sigma}_{a}^{2}}}{\hat{m}} \cdot 100
$$

i) Coeficiente de variação experimental $\left(C V_{e}\right)$ : $C V_{e}(\%)=\frac{\sqrt{\left[\left(0,75 . \hat{\sigma}_{a}^{2}+\hat{\sigma}_{e}^{2}\right) / n\right]+\hat{\sigma}_{c}^{2}}}{\hat{m}} .100 ;$ em que: $n$ número de plantas por parcela

j) Coeficiente de variação relativa $\left(C V_{r}\right)$ :

$$
C V_{r}=\frac{C V_{g p}}{C V_{e}}
$$

k) Acurácia da seleção de progênies, assumindo sobrevivência completa $\left(r_{\hat{a} a}\right)$ :

$r_{\hat{a} a}=\sqrt{\hat{h}_{m}^{2}}$

Para a análise multivariada, utilizaram-se os dados processados pelo modelo 95 , da análise univariada (Software Selegen - Reml/Blup). Na sequência, os dados foram analisados pelo método multivariada, utilizando o modelo 104 do mesmo Software, que consiste na técnica de agrupamento Tocher (Resende \& Duarte 2007). Para a separação das progênies de acordo com os caracteres, estabelecendo-se o agrupamento das progênies, utilizou-se o referido método de Tocher, considerando a distância de Mahalanobis para determinar a dissimilaridade genética e delimitação dos grupos entre as progênies. O método de Tocher é uma técnica de agrupamentos que adota o critério da distância média intragrupo e que deve ser menor que a distância intergrupo (Cruz 2008). 


\section{Resultados e Discussão}

O teste de progênies e procedência de Dipteryx alata apresentou baixa taxa de sobrevivência (55\%) se comparado com os de Saboya \& Borghetti (2012) (96\%), Ajalla et al. (2012) (93,34\%), Martimotto et al. (2012) (79\%) e Queiroz \& Firnimo (2014) (63,9\%). O principal problema para estabelecimento de plantios florestais, principalmente para os homogêneos, é a competição com braquiária (Urochloa decumbens (Stapf) R.D.Webster). Vários trabalhos ressaltam que a ausência de braquiária na fase inicial no estabelecimento de plantios de espécies arbóreas contribuiu positivamente para sobrevivência das espécies. Pires et al. (2012) também concluíram que a ausência de braquiária na fase inicial de estabelecimento de duas espécies arbóreas de leguminosas propiciaria maior sucesso. Outros fatores, como adaptação ao ambiente, competição com outras espécies e a formação do sistema radicular na ocasião do plantio também podem ter contribuído para a mortalidade das mudas. A média de sobrevivência aos 21 meses foi de 55\%, semelhante ao resultado obtido por Venturoli et al. (2011) aos 12 meses de idade.

Os valores médios de altura foram de $0,48 \mathrm{~m}$ e 1,49 $\mathrm{m}$ aos sete e 21 meses após o plantio. Apresentou um incremento médio anual de 0,82 e 0,85 , respectivamente, resultados inferiores quando comparados com os de Correa et al. (2000), que obtiveram IMA de 1,8 m com 30 dias após emergência das plântulas de Dipteryx alata; Martinotto et al. (2012), aos seis meses de idade, em consórcio com mandioca e em monocultura, com e sem adubação fosfatada, o IMA foi de 1,75 m e 1,9 m, respectivamente; Sampaio $\&$ Pinto (2007) apresentaram incremento médio de 1,56 , entre várias avaliações ocorridas entre 30 dias e seis meses após a emergência de plântulas, Canuto et al.; (2004) obtiveram IMA de 1,24, 1,17 e 1,10 em altura de plântulas, provenientes dos Estados de Mato Grosso do Sul, Minas Gerais e Goiás, respectivamente. Porém os resultados de diâmetro a altura do colo $(1,0 \mathrm{~cm})$ são superiores aos dados obtidos por Ferreira et al.; (1998) $(0,69 \mathrm{~cm})$, Corrêa (2000) $(0,45 \mathrm{~cm}) \mathrm{e}$ Canuto (2005) $(0,33 \mathrm{~cm})$.

O coeficiente de variação experimental $\left(\mathrm{CV}_{e}\right)$ variou de $28,80 \%$ até $52,36 \%$, considerado com baixa precisão por Gomes (2000), ou seja, baixo controle ambiental, com heterogeneidade dentro dos blocos. Entretanto, como se trata de um experimento com plantas no estágio juvenil e por apresentarem baixa altura, são mais suscetíveis a condições adversas do ambiente, e isso pode refletir no coeficiente de variação experimental, pode-se observar na tabela 1 que, para o mesmo caráter altura e em três períodos de avaliação, estes valores tenderam a aumentar (39,86\% a 52,36\%). No entanto, quando as plantas se estabilizarem no ambiente, este coeficiente será mais constante nos períodos de avaliações. Ledo et al. (2003) comentaram que o inconveniente dessa classificação do coeficiente de variação experimental é de não levar em consideração a cultura estudada, a variável em estudo, a heterogeneidade do solo e o tamanho da parcela, entre outros.

$\mathrm{O}$ efeito de progênies apresentou diferenças significativas a $1 \%$ de probabilidade. Para Cruz et al. (2004) a existência de variação é um fator básico ao melhoramento que subsidia o progresso genético com a prática da seleção, o que amplia a probabilidade de sobrevivência da espécie em áreas antropizadas.

$\mathrm{O}$ coeficiente de variação genética aditiva individual $\left(C V_{g i}\right)$ foi considerado alto, de 14,93\% para o DMC aos sete meses de idade a $39,88 \%$ para altura aos três meses de idade. De acordo com Aguiar et al. (2010), quanto maior o valor para o $C V_{g i}$, maior será a facilidade para encontrar indivíduos superiores que poderão proporcionar ganhos na seleção. Esses resultados sugerem que a existência de variação genética possibilita a exploração desse teste de progênies para formação de pomares de sementes por mudas e como fontes de propágulos para programas de recuperação de áreas degradadas.

O coeficiente de variação genotípico entre progênies foi inferior aos de $C V_{g p}$, variando de 7,47\% para DMC a 19,94\% para altura aos três meses de idade; valores semelhantes foram encontrados por Oliveira et al. (2006), em torno de $20 \%$ para os caracteres de comprimento total de raiz e número de raízes finas e $7 \%$ para o caráter diâmetro de raiz.

O coeficiente de herdabilidade da média de progênies $\left(\hat{h}_{m}^{2}\right)$ foi superior às estimativas dos demais coeficientes, para os caracteres altura aos três meses $(0,81)$, altura aos sete meses $(0,64)$, altura aos 21 meses e DMC $(0,53)$, fato esperado quando se assume as progênies como sendo de meiosirmãos. Esses resultados mostram o ótimo controle genético das progênies, evidenciando possíveis ganhos mediante aplicação da seleção das melhores progênies como genitores para produção de sementes que serão fornecidas para confecções de mudas para reflorestamentos.

A herdabilidade individual no sentido restrito $\left(\hat{h}_{a}^{2}\right)$, que quantifica a importância relativa da proporção 
aditiva da variância genética que pode ser transmitida para a próxima geração, apresentou intervalo de variação de 0,25 (altura aos 21 meses e DMC) a 0,80 (altura aos três meses de idade). Em todos os caracteres foram encontradas estimativas significativas para os efeitos genotípicos, pois o intervalo de variação não inclui o zero $(0,21$ para altura aos três meses, 0,18 para altura aos sete meses, 0,15 para altura aos 21 meses e DMC), o que reforça ainda mais as diferenças genéticas existentes entre os indivíduos e, portanto, a variabilidade genética. Todas as estimativas são interpretadas por Resende (1995) como medianas. Dessa forma, várias estratégias de seleção podem ser aplicadas, inclusive a aplicação da seleção massal, que é justificável quando a o valor da herdabilidade é em torno de 0,50 , como no caráter altura aos três meses de idade.
A herdabilidade para seleção dentro de progênies $\left(\hat{h}_{a d}^{2}\right)$ apresentou amplitude de variação considerável, 0,2 para altura aos 21 meses e DMC a $0,75 \%$ para altura aos três meses. $\mathrm{O}$ intervalo de variação para $\hat{h}_{a d}^{2}$ é similar a $\hat{h}_{a}^{2}$ e inferiores a $\hat{h}_{m}^{2}$, designando que a seleção com base na média de famílias deve ser mais eficiente que a seleção dentro de famílias, considerando a mesma intensidade de seleção. Segundo Resende (2002) é comum encontrar, para caracteres quantitativos, valores de baixa magnitude para herdabilidade individual e que, em geral, conduzem a estimativa de magnitude de moderada a alta, para a herdabilidade em nível de média de progênies.

Seguindo a classificação proposta por Resende \& Duarte (2007), a acurácia foi alta $\left(0,70 \leq r_{\hat{a} a}<0,90\right)$ para todos os caracteres avaliados $(0,90$ para altura

Tabela 1. Estimativas de parâmetros estatísticos e genéticos de altura total das plantas com três, sete e 21 meses, diâmetro médio do caule (DMC) aos sete meses, em um teste de progênies de Dipteryx alata Vog., proveniente da Estação Ecológica de Paulo de Faria, SP, Brasil.

Table 1. Estimates of statistical and genetic parameters of height with three (3), seven (7), and 21 months, stem diameter (DMC) at seven months, in a progeny test of Dipteryx alata Vog., from the Ecological Station of Paulo de Faria, São Paulo State, Brazil.

\begin{tabular}{|c|c|c|c|c|}
\hline \multirow{2}{*}{ Estimativas } & \multicolumn{3}{|c|}{ Altura (m) } & \multirow{2}{*}{$\frac{\mathrm{DMC}(\mathrm{cm})}{7}$} \\
\hline & 3 & 7 & 21 & \\
\hline$\overline{\hat{h}_{a}^{2}}$ & $0,80 \pm 0,21$ & $0,38 \pm 0,18$ & $0,25 \pm 0,15$ & $0,25 \pm 0,15$ \\
\hline$\hat{h}_{m}^{2}$ & 0,81 & 0,64 & 0,53 & 0,53 \\
\hline$r_{\hat{a} a}$ & 0,90 & 0,80 & 0,73 & 0,73 \\
\hline$\hat{h}_{a d}^{2}$ & 0,75 & 0,32 & 0,20 & 0,20 \\
\hline$C V_{g i}(\%)$ & 39,88 & 28,23 & 26,93 & 14,93 \\
\hline$C V_{g p}(\%)$ & 19,94 & 14,11 & 13,46 & 7,47 \\
\hline$C V_{e}(\%)$ & 39,86 & 43,47 & 52,36 & 28,80 \\
\hline$C V_{r}$ & 0,50 & 0,32 & 0,26 & 0,26 \\
\hline$\hat{m}$ & 0,25 & 0,48 & 1,49 & 1,00 \\
\hline $\operatorname{LRT}\left(\chi^{2}\right)$ & $57,33 * *$ & $10,54 * *$ & $2,12 * *$ & $4,48^{*}$ \\
\hline
\end{tabular}

** significativo a $1 \%$, *significativo a $5 \%$, com 1 grau de liberdade, ${ }^{\text {ns }}$ não significativo. $\hat{h}_{a}^{2}$ : herdabilidade individual dos efeitos aditivos; $\hat{h}_{m}^{2}$ : herdabilidade da média de progênies; $r_{\hat{a} a}:$ acurácia; $\hat{h}_{a d}^{2}:$ herdabilidade aditiva dentro de parcela; $C V_{g i}$ : coeficiente de variação genética aditiva individual; $C V_{g p}$ : coeficiente de variação genotípica entre progênies; $C V_{e}$ : coeficiente de variação experimental; $C V_{r}$ : coeficiente de variação relativa; $\hat{m}$ : média geral ; LRT: Teste da razão de verossimilhança. 
aos três meses, 0,80 para altura aos sete meses e 0,73 pra altura aos 21 meses e DMC), indicando boa precisão no acesso à variação genética verdadeira a partir da variação fenotípica observada para o caráter. Resende et al. (1995) mencionaram que a acurácia $\left(r_{\hat{a} a}\right)$ é diretamente proporcional ao ganho genético.

O coeficiente de variação relativa $(C V)$ é o parâmetro que indica a razão entre o coeficiente genotípico do indivíduo e o coeficiente experimental utilizado para estimá-lo. De acordo com Vencovsky $\&$ Barriga (1992), caracteres que possuem valores de $C V_{r}$ próximos a um são indicados para a realização de uma seleção adequada. Assim, o caráter altura aos três meses de idade $(0,50)$ é o mais indicado para resultados promissores. Porém o caráter apresentou variação com as idades de avaliação, pois até que haja um estabelecimento dos caracteres de crescimento a campo, pode haver influência do ambiente.

$\mathrm{O}$ método de agrupamento Tocher permitiu a formação de seis grupos (tabela 2), confirmando a presença de variabilidade no germoplasma e divergência entre as progênies; assim, o grupo I reuniu o maior número de progênies, demonstrando que eles são similares entre si. A similaridade nessas progênies pode ser devida à sua origem a partir de uma população ancestral comum, ou ainda, que essas populações possam ter sofrido ação antrópica, dispersão de frutos via animais ou polinização cruzada (Siqueira et al. 1993). Para realizar a coleta de sementes dentro do teste de progênies é necessária a escolha de progênies pertencentes a grupos distintos, para manter a variabilidade genética e, concomitantemente, o sucesso da restauração florestal.

Os resultados apresentados demonstram que as progênies estudadas são promissoras para a utilização em empreendimento de restauração ecológica com outras espécies arbóreas nativas, formando Áreas de Preservação Permanente, Reserva Legal, sistemas agroflorestais (SAF) e na recuperação de áreas degradadas.

Tabela 2. Formação de grupos com base no método de Tocher entre os caracteres altura total das plantas e diâmetro médio do caule em progênies de Dipteryx alata Vog. procedentes de Paulo de Faria, SP, Brasil, aos sete meses de idade, no município de Selvíria, MS, Brasil.

Table 2. Forming groups based on Tocher method between the traits height and stem diameter (DBH) in Dipteryx alata Vog. progenies from Paulo de Faria, SP, Brazil, at seven months of age, in Selvíria, MS, Brazil.

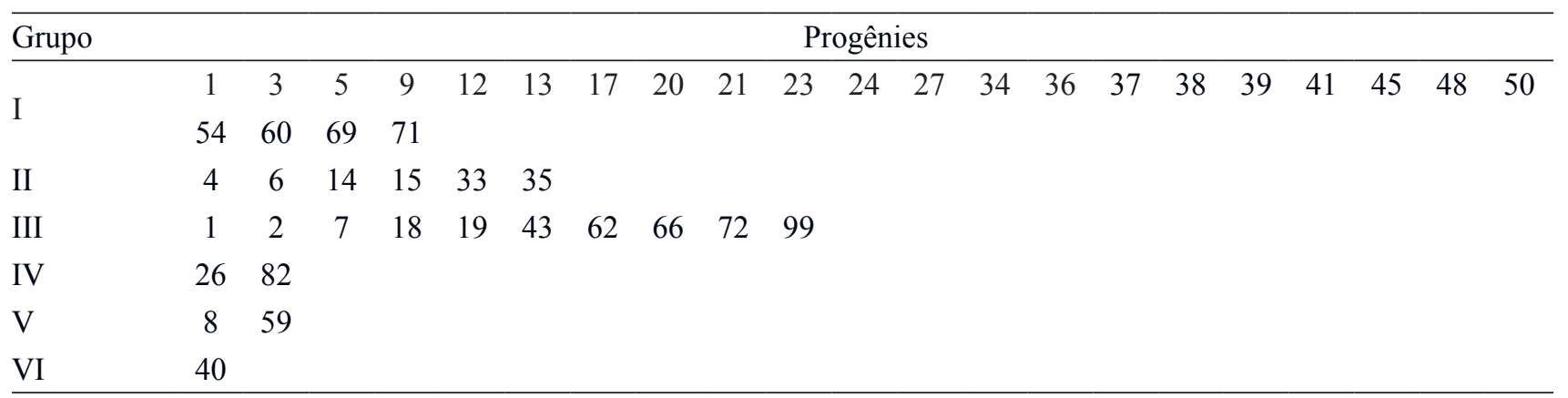

\section{Conclusões}

A formação do teste de progênies de Dipteryx alata proveniente da Estação Experimental de Paulo de Faria permitiu resgatar o material genético que ainda resta no Estado de São Paulo, com variabilidade genética suficiente para garantir a formação de novas populações.

As estimativas dos parâmetros genéticos apresentaram base genética promissora, o que permite ganhos na seleção em programas de melhoramento e a formação de pomar de sementes, adaptadas às condições edafoclimáticas da região, para utilização em programas de restauração do Cerrado.

\section{Agradecimentos}

Aos funcionários da Universidade Estadual Paulista, José Cambuim e Alonso Angelo da Silva, pela realização da coleta se sementes feita na Estação Ecológica de Paulo de Faria e pelas confecções e cuidados com as mudas. À FAPESP, pelo apoio financeiro do projeto de pesquisa processo 12/18747-2. À Pró-Reitoria de Pesquisa pela bolsa de Jovem Pesquisador. Ao CNPq pelo incentivo aos Pesquisadores MLTM e MLMF.

\section{Literatura citada}

Aguiar, A.V., Sousa, V.A., Shimizu, J.Y. 2010. Seleção genética de progênies de Pinus greggii para formação de pomares de sementes. Pesquisa Florestal Brasileira 30: $107-117$. 
Ajalla, A.C.A., Volpe, E., Vieira, M.C., Zárate, N.A.H. 2012. Produção de mudas de baru (Dipteryx alata Vog.) sob três níveis de sombreamento e quatro classes texturais de solo. Revista Brasileira de Fruticultura 34: 888-896.

Canuto, D.S.O. 2005. Variação genética entre e dentro de populações naturais de Dipteryx alata Vog. por caracteres fisiológicos e bioquímicos de sementes. Dissertação, Universidade Estadual Paulista, Ilha Solteira.

Canuto, D.S.O., Silva A.M., Cambuim, J., Moraes, M.L.T. 2004. Avaliação de altura de plântulas em três populações naturais de baru. Anais do 15 ENCONTRO DE BIÓLOGOS DO CRBio-1 (SP, MT, MS), 2004, São Pedro, pp. 121.

Corrêa, G.C., Rocha, M.R., Naves, R.V. 2000. Germinação de sementes e emergência de plântulas de baru (Dipteryx alata Vog.) no Cerrados do estado de Goiás. Pesquisa Agropecuária Tropical 17-23.

Cruz, C. D. 2008. Programa Genes: diversidade genética. Universidade Federal de Viçosa, Viçosa.

Cruz, C.D., Regazzi, A.J., Carneiro, P.C.S. 2004. Modelos biométricos aplicadas ao melhoramento genético. $3 \mathrm{ed}$. Universidade Federal de Viçosa, Viçosa.

Durigan, G., Siqueira, M.F., Franco, G.A.D.C. 2007. Threats to the Cerrado remnants of the state of São Paulo, Brazil. Scientia Agricola 64: 355-363.

Empresa Brasileira de Pesquisa Agropecuária. 1999. Sistema Brasileiro de Classificação de Solos. EMBRAPA/CNPSO, Rio de Janeiro.

Ferreira, R.A., Botelho, S.A., Davide, A.C., Malavasi, M.M. 1998. Caracterização morfológica de fruto, semente, plântula e muda de Dipteryx alata Vog. - Baru (leguminosae Papilionoideae). Cerne 4: 073-087.

Frankel, O.H., Soulé, M.S. 1981. Conservation and evolution. Cambridge University Press, Cambridge.

Gomes, F.P. 2000. Curso de Estatística Experimental. 14 ed. Degaspari, Piracicaba.

Hernandez, F.B.T., Lemos-Filho, M.A.F., Buzetti, S. 1995. Software HIDRISA e o balanço hídrico de Ilha Solteira: UNESP/FEIS - Área de Hidráulica e Irrigação. Série irrigação, 1.

Houle, D. 1992. Comparing evolvability and variability of quantitative traits. Genetics 30: 195-204.

Ledo, C.A.S., Silva, S.O., Conceição, K.S. 2003. Avaliação do coeficiente de variação na experimentação com bananeira. Anais do Simpósio Brasileiro sobre Bananicultura, Paracatu, pp.238-240.

Martinotto, F., Martinotto, C., Coelho, M.F.B., Azevedo, R.A.B., Albuquerque, M.C.F. 2012. Sobrevivência e crescimento inicial de espécies arbóreas nativas do Cerrado em consórcio com mandioca. Pesquisa Agropecuária Brasileira 47: 22-29.
Ministério do Meio Ambiente - MMA. 2015. O Bioma Cerrado. Disponível em http://www.mma.gov.br/ biomas/cerrado (acesso em 12-VIII-2015).

Oliveira, A.N., Silva, A.C., Rosado, S.C.S., Rodrigues, E.A.C. 2006. Variações genéticas para características do sistema radicular de mudas de baru (Dipteryx alata Vog.). Revista Árvore 30: 905-909.

Pires, A.C.V., Pereira, S.R., Fernandes, G.W., Oki, Y. 2012. Efeito de Brachiaria decumbens na herbivoria e no desenvolvimento de duas leguminosas nativas de cerrado. Planta Daninha 30: 737-746.

Queiroz, S.E.E., Firmino, T.O. 2014. Efeito do sombreamento na germinação e desenvolvimento de mudas de baru (Dipteryx alata Vog.). Revista Biociências 20: 64-69.

Resende, M.D.V. 1995. Delineamento de experimentos de seleção para maximização da acurácia seletiva e do progresso genético. Revista Árvore 19: 479-500.

Resende, M.D.V. 2002. Genética biométrica e estatística no melhoramento de plantas perenes. EMBRAPA Informação Tecnológica, Brasília.

Resende, M.D.V. 2007 a. Matemática e estatística na análise de experimentos e no melhoramento genético. EMBRAPA Florestal, Colombo.

Resende, M.D.V. 2007b. Selegen -Reml/Blup: sistema estatístico e seleção genética computadorizada via modelos lineares mistos. EMBRAPA Florestas, Colombo.

Resende, M.D.V., Duarte, J.B. 2007. Precisão e controle experimental de qualidade em experimentos de avaliação de cultivares. Pesquisa Agropecuária Tropical 37: 182-194.

Saboya, P., Borghetti, F. 2012. Gremination, initial growth, and biomass allocation in three native Cerrado species. Brazilian Journal of Botany 35: 129-135.

Sampaio, J.C., Pinto, J.R.R. 2007. Critérios para avaliação do desempenho de espécies nativas lenhosas em plantios de restauração no Cerrado. Revista Brasileira de Biociências 51: 504-506.

Siqueira, C.M.F., Nogueira, J.C.B., Kageyama, P.Y. 1993. Conservação dos recursos genéticos ex situ do cumbaru Dipteryx alata Vog. - Leguminosae. Revista do Instituto Florestal 5: 231-243.

Stranghetti, V., Ranga, N.T. 1998. Levantamento florístico das espécies vasculares da floresta estacional mesófila semidecídua da Estação Ecológica de Paulo de Faria SP. Revista Brasileira de Botânica 21: 3 Disponível em http://dx.doi.org/10.1590/S0100-84041998000300008 (acesso em 15-IX-2015).

Takemoto, E., Okada, I.A., Garbelotti, M.L., Tavares, M., Aude-Pimentel,S. 2001. Composição química da semente e do óleo de baru (Dipteryx alata Vog.) nativo do município de Pirinópolis, estado de Goiás. Revista do Instituto Adolfo Lutz 60: 113-117. 
Togashi, M. 1993. Composição e caracterização química e nutricional do fruto do baru (Dipteryx alata Vog.). Dissertação, Universidade Estadual de Campinas, Campinas.

Vallilo, M.I., Tavares, M., Aude-Pimentel, S., Garbelotti, M.L., Campos, N.C. 2001. Composição química e o perfil de ácidos graxos das sementes de quatro espécies de palmeira cultivadas no estado de São Paulo. Revista do Instituto Florestal 13: 147-154.

Vencovsky, R., Barriga, P. 1992. Genética biométrica no fitomelhoramento. Sociedade Brasileira de Genética, Ribeirão Preto.
Venturoli, F., FAGG, C.W., FELFILI, J.M. 2011. Desenvolvimento inicial de Dipteryx alata Vog. e Myracrodruon urundeuva Allemão em plantio de enriquecimento de uma floresta estacional semidecídua secundária. Bioscience Journal 27: 482-493.

Vera, R., Soares Junios, M.S.S., Naves, R.V., Souza, E.B.S., Fernandes, E.P., Caliari, M., Leandro, W.M. 2009. Características químicas de amêndoas de barueiros (Dipteryx alata Vog.) de ocorrência natural no Cerrado do Estado de Goiás, Brasil. Revista Brasileira de Fruticultura 31: 112-118. 